(C) [2007] IEEE. Reprinted, with permission, from [Li Li, A Gramian-based approach to model reduction for uncertain systems, Decision and Control, 2007 46th IEEE Conference on, 12-14 Dec. 2007]. This material is posted here with permission of the IEEE. Such permission of the IEEE does not in any way imply IEEE endorsement of any of the University of Technology, Sydney's products or services. Internal or personal use of this material is permitted.

However, permission to reprint/republish this material for advertising or promotional purposes or for creating new collective works for resale or redistribution must be obtained from the IEEE by writing to pubs-permissions@ieee.org. By choosing to view this document, you agree to all provisions of the copyright laws protecting it 


\title{
A Gramian-based Approach to Model Reduction for Uncertain Systems
}

\author{
Li Li, Ian R. Petersen
}

\begin{abstract}
The paper considers the problem of model reduction for a class of uncertain systems with structured norm bounded uncertainty. The paper introduces controllability and observability Gramians in terms of certain parameterized algebraic Riccati inequalities. This enables a balanced truncation model reduction procedure for uncertain systems to be presented. Error bounds for this model reduction procedure are derived. The paper also investigates $H_{\infty}$ model reduction for uncertain systems. The solution to this problem is shown to involve constructing the underlying Gramians satisfying a certain rank constraint.
\end{abstract}

\section{INTRODUCTION}

Model reduction is an important aspect of linear systems theory. One of the most commonly applied methods is the balanced truncation method; e.g., see [1]. This method involves finding the controllability and observability Gramians for the system under consideration. Then a state space transformation is found which simultaneously diagonalizes the Gramians. This leads to a balanced realization of the system from which the reduced order model is constructed together with an a priori error bound; e.g., see [2], [3], [4].

Uncertain systems commonly arise in robust control theory; e.g., see [5], [6]. Model reduction methods for uncertain systems are very useful in the design of practical robust control systems in which the dimension of the controller needs to be limited. For the discrete-time case, the balanced truncation method for uncertain systems can be traced back to [7] within the framework of linear fractional transformations (LFTs). This technique was further developed in [8] for multidimensional and uncertain systems. In [9], [10], balanced model reduction was extended to linear time-varying system. [11] considered model reduction for the class of nonlinear systems described by a discrete-time state equation containing a repeated scalar nonlinearity. For the continuoustime case, [12], [13] considered the gain scheduling problem for uncertain systems described by an LFT representation, and derived some linear matrix inequality (LMI) conditions to guarantee the existence of gain-scheduled controllers. This included model reduction as a special case of these problems. Model reduction for linear parameter-varying systems was proposed in [14], [15], [16], [17]. Closely related problems, such as approximation, truncation and simplification of uncertain systems were presented in [18], [19].

This work was supported by the Australian Research Council.

L. Li is with National ICT Australia, Department of Electrical and Electronic Engineering, The University of Melbourne, VIC, 3010, Australia li.lidee. unimelb.edu.au.

I. R. Petersen is with School of Information Technology and Electrical Engineering, University of New South Wales at the Australian Defence Force Academy, Canberra ACT 2600, Australia, irp@ee.adfa.edu.au.
In the recent papers [20], [21], problems of controllability and unobservability were investigated for a class of structured uncertain systems in which the uncertainty is described by Integral Quadratic Constraints (IQCs). These results motivate the question as to whether a balanced truncation method can be obtained for uncertain systems. In this paper, we study the balancing model reduction problem for continuous-time uncertain systems modeled by an LFT representation, as a counterpart to the corresponding results for discrete-time uncertain systems [7], [8]. We consider uncertain systems with norm bounded uncertainty rather than the IQC uncertainty description considered in [22], [20], [21]. This enables us to develop a balanced truncation method for the class of uncertain systems under consideration. Gramians and related realization theory topics for discrete-time uncertain systems were discussed in [23], [24], [25].

In this paper, two parameterized algebraic Riccati inequalities (ARIs) are introduced to characterize the controllability and observability of the uncertain systems under consideration. These results are closely related to those in [20], [21]. We focus on the observability property rather than unobservability as in [20], and thus a different ARI is used to account for this. A sufficient and necessary condition to guarantee the feasibility of the two ARIs are provided. It is proved that the solutions to these ARIs are controllability and observability Gramians of the uncertain systems. Based on these results, we present a balanced truncation algorithm to reduce the dimension of the uncertain systems. Our proposed method is based on the use of Linear Matrix Inequalities (LMIs) to construct the desired reduced dimension uncertain system model. Our main results give bounds on the $H_{\infty}$ norm error introduced by the model reduction process. The first cost bound obtained is of the same form as the bound which arises in the standard balanced truncation approach. This bound applies to the case in which the same uncertainty realization is considered in both the original and reduced dimension uncertain systems; see Theorem 11. The second bound obtained applies to the case in which different uncertainties are allowed in the original and reduced order uncertain system. This bound is of a similar form to the first bound but an extra term is introduced to allow for the different uncertainties in the two systems; see Theorem $14 . H_{\infty}$ model reduction for uncertain systems is also investigated, and a sufficient condition for the existence of a reduced order model is provided which involves the underlying Gramians together with a rank constraint; see Theorem 16. Similar $H_{\infty}$ model reduction results can also be found in [16] for polytopic uncertain linear systems. 
Notation Let $\mathbf{L}_{2}^{m}[0, \infty)$ be the space of square integrable functions in $\mathbf{R}^{m}$, and $\mathcal{L}\left(\mathbf{L}_{2}^{m}\right)$ denote the space of all linear bounded operators mapping from $\mathbf{L}_{2}^{m}$ to $\mathbf{L}_{2}^{m}$. The gain of an operator $\Delta$ in $\mathcal{L}\left(\mathbf{L}_{2}^{m}\right)$ is given by $\|\Delta\|=\sup _{z \in \mathbf{L}_{2}^{m}[0, \infty), z \neq 0} \frac{\|z\|}{\|z\|}$, and the adjoint operator of $\Delta$ is denoted as $\Delta^{*}$ if $\Delta$ is linear, and if $\Delta=\Delta^{*}, \Delta<0$ means that $x^{*} \Delta x<0$ for any $x \neq 0$ in $\mathbf{R}^{m}$. We also use $M^{*}$ to denote the complex conjugate transpose of a complex matrix $M$. For $z \in \mathbf{R}^{m}$ and a nonnegative matrix $\Lambda \in \mathbf{R}^{m \times m},|z|_{\Lambda}^{2}=z^{*} \Lambda z$, and $\Lambda$ is omitted when it is an identity matrix. The state-space realization of a transfer matrix is denoted by $G(s)=\left[\begin{array}{l|l}A & B \\ \hline C & D\end{array}\right]:=C(s I-A)^{-1} B+D$.

\section{PROBLEM Formulation}

We consider the uncertainty structure $\Delta^{\boldsymbol{c}}=\left\{\operatorname{diag}\left(\Delta_{1}, \cdots, \Delta_{k}\right): \Delta_{i} \in \mathcal{L}\left(\mathbf{L}_{2}^{h_{i}}\right), \Delta_{i}\right.$ causal, $\left.\left\|\Delta_{i}\right\| \leq 1\right\}$, and the following uncertain system:

$$
\mathcal{G}_{\Delta}:\left\{\begin{array}{l}
\dot{x}=A x+E \xi+B u, \\
z=K x+G u, \\
y=C x+D \xi, \\
\xi=\Delta z, \quad \Delta \in \Delta^{c},
\end{array}\right.
$$

where $x \in \mathbf{R}^{n}$ is the state, $u \in \mathbf{R}^{m}$ is the control input, $z \in \mathbf{R}^{h}$ is the uncertainty output, $y \in \mathbf{R}^{l}$ is the measured output and $\xi \in \mathbf{R}^{h}$ is the uncertainty input; here $h=h_{1}+\cdots+h_{k}$.

Let the nominal system be denoted by

$$
M=\left[\begin{array}{ll}
M_{11} & M_{12} \\
M_{21} & M_{22}
\end{array}\right]=\left[\begin{array}{c|cc}
A & E & B \\
\hline K & \mathbf{0}_{h} & G \\
C & D & \mathbf{0}_{l \times m}
\end{array}\right] .
$$

Then, the uncertain system (1) is defined by an LFT representation as follows. For any bounded linear operator $\Delta \in \mathcal{L}\left(\mathbf{L}_{2}^{h}\right)$ such that $I-M_{11} \Delta$ is non-singular, define

$$
\mathcal{F}_{u}(M, \Delta):=M_{22}+M_{21} \Delta\left(I-M_{11} \Delta\right)^{-1} M_{12} .
$$

Define the following operators

$$
\left[\begin{array}{ll}
\mathcal{A}_{\Delta} & \mathcal{B}_{\Delta} \\
\mathcal{C}_{\Delta} & \mathcal{D}_{\Delta}
\end{array}\right]=\left[\begin{array}{cc}
A+E \Delta K & B+E \Delta G \\
C+D \Delta K & D \Delta G
\end{array}\right] .
$$

If $\Delta$ varies in the class $\Delta_{R C}$ of real constant matrices of the underlying structure, the uncertain system (1) corresponds to

$$
\mathcal{G}_{\Delta}=\mathcal{F}_{u}(M, \Delta)=\left[\begin{array}{c|c}
\mathcal{A}_{\Delta} & \mathcal{B}_{\Delta} \\
\hline \mathcal{C}_{\Delta} & \mathcal{D}_{\Delta}
\end{array}\right], \Delta \in \Delta_{R C} .
$$

Robust stability of the uncertain system (1) is defined below. It is assumed that the system (1) is robustly stable throughout this paper. As in the traditional balanced truncation, this assumption is essential for the balanced truncation of the uncertain system (1), and guarantees the existence of the solutions to certain ARIs arising from underlying controllability and observability problems; see Theorem 5.

Definition 1 (Robust Stability [26]): The uncertain system (1) is robustly stable if $\left(I-M_{11} \Delta\right)^{-1}$ exists in $\mathcal{L}\left(\mathbf{L}_{2}^{n}\right)$ and is causal, for all $\Delta \in \Delta^{c}$.
The following lemma states a necessary and sufficient condition for robust stability. It is given in terms of the positive commutant set corresponding to $\Delta^{\boldsymbol{c}}$ defined as

$$
\boldsymbol{P}_{\boldsymbol{\Theta}}=\left\{\operatorname{diag}\left(\theta_{1} I_{h_{1}}, \cdots, \theta_{k} I_{h_{k}}\right): \theta_{i}>0\right\} .
$$

Lemma 2: (see [26]) The system (1) is robustly stable if and only if there exist $\Theta \in \boldsymbol{P}_{\boldsymbol{\Theta}}$ and $X>0$, such that

$$
A^{*} X+X A+K^{*} \Theta K+X E \Theta^{-1} E^{*} X<0 .
$$

\section{CONTRollability AND OBSERVABILITy GRAMIANS}

As is well known, the controllability and observability Gramians play very important roles in the traditional balanced truncation approach to model reduction; see [1]. In this section, we introduce Gramians for the uncertain system (1). First the controllability and observability Gramians for the uncertain system (1) are defined as follows.

Definition 3: A matrix $S>0$ is said to be a generalized controllability Gramian for the uncertain system (1) if the following linear, operator inequality holds,

$$
\mathcal{A}_{\Delta} S+S \mathcal{A}_{\Delta}^{*}+\mathcal{B}_{\Delta} \mathcal{B}_{\Delta}^{*}<0 \quad \forall \Delta \in \Delta^{\boldsymbol{c}} .
$$

Similarly, a matrix $P>0$ is said to be a generalized observability Gramian for the uncertain system (1) if

$$
\mathcal{A}_{\Delta}^{*} P+P \mathcal{A}_{\Delta}+\mathcal{C}_{\Delta}^{*} \mathcal{C}_{\Delta}<0 \quad \forall \Delta \in \Delta^{\boldsymbol{c}} .
$$

Here, $\mathcal{A}_{\Delta}, \mathcal{B}_{\Delta}, \mathcal{C}_{\Delta}$ are as defined in (2).

In [21], [20], issues of robust controllability and unobservability for uncertain linear systems with structured uncertainty were discussed in the framework of IQCs and the $S$-procedure. In these references, a linear time varying system with nonlinear uncertainties was studied, and certain parameterized Riccati differential equations were derived to characterize the robust controllability and unobservability of the underlying uncertain system. In this paper, we will extend these ideas to uncertain systems of the form (1), that is, we consider an LTI system with linear norm bounded uncertainties. Particularly, we focus on the observability rather than the unobservability in [20], and thus a corresponding Riccati representation is used to account for this.

Consider the following Riccati inequalities:

$$
\begin{aligned}
A S+S A^{*} & +\left(S K^{*}+B G^{*}\right)\left(\Lambda_{c}^{-1}-G G^{*}\right)^{-1}\left(K S+G B^{*}\right) \\
& +E \Lambda_{c}^{-1} E^{*}+B B^{*}<0, \\
A^{*} P+P A & +\left(P E+C^{*} D\right)\left(\Lambda_{o}-D^{*} D\right)^{-1}\left(E^{*} P+D^{*} C\right) \\
& +K^{*} \Lambda_{o} K+C^{*} C<0,
\end{aligned}
$$

where $S>0, P>0$, and $\Lambda_{c} \in \boldsymbol{P}_{\boldsymbol{\Theta}}, \Lambda_{o} \in \boldsymbol{P}_{\boldsymbol{\Theta}}$ are such that $\Lambda_{c}^{-1}-$ $G G^{*}>0, \Lambda_{o}-D^{*} D>0$. Alternatively, (7) and (8) can be rewritten as

$$
\begin{aligned}
& A S+S A^{*}+S K^{*} \Lambda_{c} K S+E \Lambda_{c}^{-1} E^{*} \\
& +\left(B+S K^{*} \Lambda_{c} G\right)\left(I_{m}-G^{*} \Lambda_{c} G\right)^{-1}\left(B^{*}+G^{*} \Lambda_{c} K S\right)<0 \\
& A^{*} P+P A+P E \Lambda_{o}^{-1} E^{*} P+K^{*} \Lambda_{o} K \\
& +\left(C^{*}+P E \Lambda_{o}^{-1} D^{*}\right)\left(I_{l}-D \Lambda_{o}^{-1} D^{*}\right)^{-1}\left(C+D \Lambda_{o}^{-1} E^{*} P\right)<0 .
\end{aligned}
$$


The following example show that $S$ in (7) or (9) is analogous to the traditional controllability Gramian for linear time-invariant systems. Similar result also hold for the observability Gramian.

Observation 4: (Controllability Gramian) Consider the uncertain system (1) on the interval $(-\infty, 0]$ with $x(-\infty)=0$, and assume that the ARI (9) admits a solution $S>0$ for some $\Lambda_{c} \in \boldsymbol{P}_{\boldsymbol{\Theta}}$ such that $I_{m}-G^{*} \Lambda_{c} G>0$.

Using $x^{*}(t) S^{-1} x(t)$ as a candidate Lyapunov function, from (9) we have

$$
\begin{aligned}
& \int_{-\infty}^{0}|u|^{2} d t \\
& \geq x_{0}^{*} S^{-1} x_{0}+\int_{-\infty}^{0}\left(|z|_{\Lambda_{c}}^{2}-|\xi|_{\Lambda_{c}}^{2}\right) d t+\int_{-\infty}^{0}\left|\xi-\Lambda_{c}^{-1} E^{*} S^{-1} x\right|_{\Lambda_{c}}^{2} d t \\
& \quad+\int_{-\infty}^{0}\left|u-\left(I_{m}-G^{*} \Lambda_{c} G\right)^{-1}\left(B^{*} S^{-1}+G^{*} \Lambda_{c} K\right) x\right|_{\left(I_{m}-G^{*} \Lambda_{c} G\right)}^{2} d t \\
& \geq x_{0}^{*} S^{-1} x_{0} .
\end{aligned}
$$

Therefore,

$$
\min \int_{-\infty}^{0}|u|^{2} d t \geq x_{0}^{*} S^{-1} x_{0}
$$

Here the minimum is respect to both the control input $u$ and the uncertainty input $\xi$.

We will show that the solutions to (7-8) or (9-10) are closely related to the controllability and observability Gramians for the uncertain system (1). Before doing that, it is necessary to address the feasibility of these inequalities.

Theorem 5: The following statements are equivalent: (i)

1) The uncertain system (1) is robustly stable.

2) The Riccati inequality (7) admits a solution $S>0$ for some $\Lambda_{c} \in \boldsymbol{P}_{\boldsymbol{\Theta}}$.

3) The Riccati inequality (8) admits a solution $P>0$ for some $\Lambda_{o} \in \boldsymbol{P}_{\boldsymbol{\Theta}}$.

Proof: We only prove the equivalence between $(i)$ and (ii). The equivalence between $(i)$ and (iii) proceeds in a similar fashion.

$(i i) \Rightarrow(i)$ : It follows directly from (9) that (4) holds for $X=S^{-1}, \Theta=\Lambda_{c}$. Then $(i)$ follows using Lemma 2 .

$(i) \Rightarrow(i i)$ : Using Lemma 2, it follows that (4) holds. Then we can choose $\varepsilon>0$ sufficiently small, such that $\varepsilon^{-1} I_{m}-$ $G^{*} \Theta G>0$ and

$$
\begin{aligned}
& A^{*} X+X A+K^{*} \Theta K+X E \Theta^{-1} E^{*} X \\
& +\left(X B+K^{*} \Theta G\right)\left(\varepsilon^{-1} I_{m}-G^{*} \Theta G\right)^{-1}\left(B^{*} X+G^{*} \Theta K\right)<0
\end{aligned}
$$

Letting $X=(\varepsilon S)^{-1}, \Theta=\varepsilon^{-1} \Lambda_{c}$, from (11), it is not difficult to derive (9), thus (7) holds.

The following theorem relates (7) and (8) to generalized controllability and observability Gramians of the uncertain system (1), as defined in Definition 3.

Theorem 6: If there exist $S>0, P>0, \Lambda_{c} \in \boldsymbol{P}_{\boldsymbol{\Theta}}, \Lambda_{o} \in \boldsymbol{P}_{\boldsymbol{\Theta}}$ solving ARIs (7), (8), then $S, P$ are generalized controllability and observability Gramians of the uncertain system (1).
Proof: We only prove the controllability part.

$$
\begin{aligned}
& \mathcal{A}_{\Delta} S+S \mathcal{A}_{\Delta}^{*}+\mathcal{B}_{\Delta} \mathcal{B}_{\Delta}^{*} \\
=(A & +E \Delta K) S+S(A+E \Delta K)^{*}+(B+E \Delta G)(B+E \Delta G)^{*} \\
= & A S+S A^{*}+E \Delta \Lambda_{c}^{-1} \Delta^{*} E^{*}+B B^{*} \\
& +\left(S K^{*}+B G^{*}\right)\left(\Lambda_{c}^{-1}-G G^{*}\right)^{-1}\left(K S+G B^{*}\right) \\
& -\left[S K^{*}+B G^{*}-E \Delta\left(\Lambda_{c}^{-1}-G G^{*}\right)\right]\left(\Lambda_{c}^{-1}-G G^{*}\right)^{-1} \\
& \times\left[S K^{*}+B G^{*}-E \Delta\left(\Lambda_{c}^{-1}-G G^{*}\right)\right]^{*} .
\end{aligned}
$$

Note that

$$
E \Delta \Lambda_{c}^{-1} \Delta^{*} E^{*}=E \Lambda_{c}^{-1 / 2} \Delta \Delta^{*} \Lambda_{c}^{-1 / 2} E^{*} \leq E \Lambda_{c}^{-1} E^{*} .
$$

Therefore, combining (12), (13), (7) and $\Lambda_{c}^{-1}-G G^{*}>0$, we conclude that (5) holds.

\section{BALANCED TRUNCATION}

As seen in the last section, solutions to the ARIs (7) and (8) are generalized Gramians for the uncertain system $\mathcal{G}_{\Delta}$ in (1). Consequently, the traditional balanced truncation technique for model reduction can be applied here. We are now ready to present our main results. First, we provide a numerical way to solve the ARIs (7) and (8). By using the Schur complement and letting $\bar{\Lambda}_{c}=\Lambda_{c}^{-1}$, (7) and (8) can be transformed into LMIs, stated in the following propositions.

Proposition 7: If there exist $S>0$ and $\bar{\Lambda}_{c} \in \boldsymbol{P}_{\boldsymbol{\Theta}}$ solving the following Semi-Definite Programming (SDP) problem:

$$
\begin{aligned}
& \text { minimize trace }(S) \text { : } \\
& {\left[\begin{array}{ccc}
S A^{*}+A S+E \bar{\Lambda}_{c} E^{*} & S K^{*} & B \\
\star & -\bar{\Lambda}_{c} & G \\
\star & \star & -I_{m}
\end{array}\right]<0,}
\end{aligned}
$$

then $S$ is a generalized controllability Gramian for the uncertain system (1). Here the notation $\star$ in the above matrix indicates that the corresponding elements in the matrix are such that the overall matrix is symmetric.

Proposition 8: If there exist matrices $P>0$ and $\Lambda_{o} \in \boldsymbol{P}_{\boldsymbol{\Theta}}$ solving the following SDP problem:

$$
\begin{aligned}
& \operatorname{minimize} \operatorname{trace}(P) \text { : } \\
& {\left[\begin{array}{ccc}
A^{*} P+P A+K^{*} \Lambda_{o} K & P E & C^{*} \\
\star & -\Lambda_{o} & D^{*} \\
\star & \star & -I_{l}
\end{array}\right]<0,}
\end{aligned}
$$

then $P$ is a generalized observability Gramian for the uncertain system (1).

Definition 9: An uncertain system of the form (1) is said to be balanced if it has generalized observability and controllability Gramians which are identical diagonal matrices.

We summarize the proposed model reduction algorithm as follows.

Procedure 10:

1) Solve SDP problems (14) and (15) to obtain generalized Gramians $S>0, P>0$.

2) Balance $S, P$ by constructing a state transformation matrix $T[3]$ such that

$$
\begin{aligned}
T S T^{*} & =\left(T^{-1}\right)^{*} P T^{-1} \\
& =\Sigma=\operatorname{diag}\left(\Sigma_{1}, \Sigma_{2}\right)=\operatorname{diag}\left(\gamma_{1}, \ldots, \gamma_{n}\right)
\end{aligned}
$$


where $\gamma_{1} \geq \ldots \geq \gamma_{d}>\gamma_{d+1} \geq \ldots \geq \gamma_{n}>0, \quad \Sigma_{1}=$ $\operatorname{diag}\left(\gamma_{1}, \ldots, \gamma_{d}\right), \Sigma_{2}=\operatorname{diag}\left(\gamma_{d+1}, \ldots, \gamma_{n}\right)$. The quantities $\gamma_{1}, \ldots, \gamma_{n}$ are referred to as generalized Hankel singular values for the uncertain system.

3) Write the transformed nominal system of (1) as

$$
M=\left[\begin{array}{c|cc}
\bar{A} & \bar{E} & \bar{B} \\
\hline K & \mathbf{0}_{h} & G \\
\bar{C} & D & \mathbf{0}_{l \times m}
\end{array}\right]
$$

where

$$
\bar{A}=T A T^{-1} ; \bar{E}=T E ; \bar{B}=T B ; \bar{C}=C T^{-1} ; \bar{K}=K T^{-1} .
$$

The sub-matrices of this matrix $M$ corresponding to the matrix $\Sigma_{2}$ in (16) are truncated to obtain the reduced order uncertain system defined by

$$
M_{r}=\left[\begin{array}{c|cc}
\bar{A}_{r} & \bar{E}_{r} & \bar{B}_{r} \\
\hline K_{r} & \mathbf{0}_{h} & G \\
\bar{C}_{r} & D & \mathbf{0}_{l \times m}
\end{array}\right]
$$

with order $d$.

4) Write the reduced dimension uncertain system as $\mathcal{G}_{r \Delta}=\mathcal{F}_{u}\left(M_{r}, \Delta\right), \Delta \in \Delta^{c}$.

Theorem 11: Consider the uncertain system (1) and suppose that the reduced dimension uncertain system $\mathcal{G}_{r \Delta}$ is obtained as described in Procedure 10. Then $\mathcal{G}_{r \Delta}$ is also balanced and robustly stable. Furthermore,

$$
\sup _{\Delta \in \Delta^{c}}\left\|\mathcal{G}_{\Delta}(s)-\mathcal{G}_{r \Delta}(s)\right\|_{\infty} \leq 2\left(\gamma_{1}^{\dagger}+\cdots+\gamma_{q}^{\ddagger}\right),
$$

where $\gamma_{i}^{t}$ denote the distinct generalized Hankel singular values of $\gamma_{d+1}, \ldots, \gamma_{n}$, that is, $\gamma_{1}^{t}>\gamma_{2}^{t}>\ldots>\gamma_{q}^{t}$ and $\left\{\gamma_{d+1}, \ldots, \gamma_{n}\right\}=\left\{\gamma_{1}^{t}, \ldots, \gamma_{q}^{t}\right\}$.

Proof: Here we will only prove the case when $\Delta$ is in the class $\Delta_{R C}$ of real constant matrices of the underlying structure. For a more general case when $\Delta \in \Delta^{c}$, the proof is analogous to that of Theorem 14.

Partition $M$ in (17) accordingly as

$$
M=\left[\begin{array}{cc|cc}
\bar{A}_{r} & \bar{A}_{12} & \bar{E}_{r} & \bar{B}_{r} \\
\bar{A}_{21} & \bar{A}_{22} & \bar{E}_{2} & \bar{B}_{2} \\
\hline K_{r} & K_{2} & \mathbf{0}_{h} & G \\
\bar{C}_{r} & \bar{C}_{2} & D & \mathbf{0}_{l \times m}
\end{array}\right]
$$

Then it follows from Theorem 6 that $\Sigma=\operatorname{diag}\left(\Sigma_{1}, \Sigma_{2}\right)$ is the balanced Gramian of the uncertain system

$$
\mathcal{F}_{u}(M, \Delta)=\left[\begin{array}{cc|c}
\bar{A}_{r}+\bar{E}_{r} \Delta \bar{K}_{r} & \bar{A}_{12}+\bar{E}_{r} \Delta \bar{K}_{2} & \bar{B}_{r}+\bar{E}_{r} \Delta G \\
\bar{A}_{21}+\bar{E}_{2} \Delta \bar{K}_{r} & \bar{A}_{22}+\bar{E}_{2} \Delta \bar{K}_{2} & \bar{B}_{2}+\bar{E}_{2} \Delta G \\
\hline C_{r}+D \Delta \bar{K}_{r} & C_{2}+D \Delta \bar{K}_{2} & D \Delta G
\end{array}\right] .
$$

The reduced uncertain system $\mathcal{G}_{r \Delta}$ corresponding to $\Sigma_{1}$ is

$$
\mathcal{F}_{u}\left(M_{r}, \Delta\right)=\left[\begin{array}{c|c}
\bar{A}_{r}+\bar{E}_{r} \Delta \bar{K}_{r} & \bar{B}_{r}+\bar{E}_{r} \Delta G \\
\hline C_{r}+D \Delta \bar{K}_{r} & D \Delta G
\end{array}\right],
$$

where $M_{r}$ is defined in (18). It is easy to show that $\mathcal{G}_{r \Delta}$ satisfies (7) and (8) with balanced Gramian $\Sigma_{1}$. Therefore, $\mathcal{G}_{r \Delta}$ is balanced from Theorem 6, and robustly stable from Theorem 5. The bound in (19) holds from the traditional balanced truncation technique; e.g. see [26].

In the above theorem, we assume that the original system and the reduced system have identical uncertainties. From this, it follows that the D-matrix of the error system $G_{\Delta}(s)-$
$\mathcal{G}_{r \Delta}(s)$ is canceled. Therefore, an error bound of the form (19) can be attained. If different uncertainties are allowed, the error bound will require an additional term $\bar{\theta}$ determined by $\bar{\Lambda}_{c}, \Lambda_{o}$, as to be shown below.We need the following results from [18] in the context of IQCs to provide such a bound.

Definition 12: Let $\Pi: i \mathbf{R} \rightarrow \mathbf{C}^{2 n \times 2 n}$ be a bounded measurable function, taking Hermitian values. The operator $\Delta$ : $\mathbf{C}^{n} \rightarrow \mathbf{C}^{n}$ is said to satisfy the IQC defined by the multiplier $\Pi$ if for all vectors $z, x=\Delta z$ in $\mathbf{L}_{2}^{n}[0,+\infty)$, their frequency signals $\hat{z}(i \omega), \hat{x}(i \omega)$ satisfy

$$
\int_{-\infty}^{+\infty}\left[\begin{array}{l}
\hat{z}(i \omega) \\
\hat{x}(i \omega)
\end{array}\right]^{*} \Pi(i \omega)\left[\begin{array}{l}
\hat{z}(i \omega) \\
\hat{x}(i \omega)
\end{array}\right] d \omega \geq 0
$$

Lemma 13: [18] Assume that both $\mathcal{F}_{u}(\bar{M}, \bar{\Delta})$ and $\mathcal{F}_{u}(\bar{M}, \hat{\Delta})$ are robustly stable and operators

$$
\bar{\Delta}=\operatorname{diag}\left(\bar{\Delta}_{1}, \cdots, \bar{\Delta}_{\bar{k}}\right), \quad \hat{\Delta}=\operatorname{diag}\left(\bar{\Delta}_{1}, \cdots, \bar{\Delta}_{\hat{k}}, \hat{\Delta}_{\hat{k}+1}, \cdots, \hat{\Delta}_{\bar{k}}\right)
$$

are linear. Also assume that $\bar{\Delta}_{i}$ satisfies the IQC defined by $\Pi_{i}$ for $i=1, \cdots, \bar{k}$, and $\hat{\Delta}_{i}$ satisfies the IQC defined by $\Pi_{i}$ for $i=\hat{k}+1, \cdots, \bar{k}$. Let $\Pi=\left[\begin{array}{ll}\Pi_{(1,1)} & \Pi_{(1,2)} \\ \Pi_{(2,1)} & \Pi_{(2,2)}\end{array}\right]$ be defined as

$$
\Pi_{(i, j)}=\operatorname{diag}\left(\Pi_{1(i, j)}, \cdots, \Pi_{\bar{k}(i, j)}\right), i, j=1,2,
$$

where $\Pi_{v}=\left[\begin{array}{ll}\Pi_{v(1,1)} & \Pi_{v(1,2)} \\ \Pi_{v(2,1)} & \Pi_{v(2,2)}\end{array}\right]$ for $v=1, \cdots, \bar{k}$, and assume

$$
\left[\begin{array}{cc}
\bar{M}_{11} & \bar{M}_{12} \\
I & 0
\end{array}\right]^{*} \Pi\left[\begin{array}{cc}
\bar{M}_{11} & \bar{M}_{12} \\
I & 0
\end{array}\right]<\left[\begin{array}{ll}
0 & 0 \\
0 & I
\end{array}\right]
$$

for all $\omega \in[0, \infty]$. If there exists a real matrix $\bar{\Sigma}=$ $\operatorname{diag}\left(\sigma_{1} I_{n_{1}}, \cdots, \sigma_{\bar{k}} I_{n_{\bar{k}}}\right)>0$ compatible with $\bar{\Delta}, \hat{\Delta}$ such that

$$
\left[\begin{array}{c}
\bar{M}_{11} \\
I
\end{array}\right]^{*}\left[\begin{array}{cc}
\bar{\Sigma}^{2} & 0 \\
0 & \bar{\Sigma}^{2}
\end{array}\right] \Pi\left[\begin{array}{c}
\bar{M}_{11} \\
I
\end{array}\right]+\bar{M}_{21}^{*} \bar{M}_{21}<0
$$

for all $\omega \in[0, \infty]$, then

$$
\left\|\mathcal{F}_{u}(\bar{M}, \bar{\Delta})-\mathcal{F}_{u}(\bar{M}, \hat{\Delta})\right\| \leq 2\left(\sigma_{\hat{k}+1}+\cdots+\sigma_{\bar{k}}\right) .
$$

Theorem 14: Consider the uncertain system (1) and suppose that the reduced dimension uncertain system $\mathcal{G}_{r \Delta}$ is obtained as described in Procedure 10. Then

$$
\sup _{\tilde{\Delta}, \Delta \in \Delta^{c}}\left\|\mathcal{G}_{\tilde{\Delta}}(s)-\mathcal{G}_{r \Delta}(s)\right\|_{\infty} \leq 2\left(\gamma_{1}^{t}+\cdots+\gamma_{l}^{\dagger}+\bar{\theta}\right),
$$

where $\bar{\theta}=\sum_{i=1}^{k} \sqrt{\theta_{o i} \bar{\theta}_{c i}}$, and $\theta_{o i}, \bar{\theta}_{c i}$ are repeated entries of $\Lambda_{o}, \bar{\Lambda}_{c}$ respectively, as defined in (3).

Proof: For any $\tilde{\Delta}, \Delta \in \boldsymbol{\Delta}^{\boldsymbol{c}}$, let the parameters in Lemma 13 be defined as follows:

$$
\begin{gathered}
\hat{k}=1, \bar{k}=3, \Pi_{1}=\left[\begin{array}{cc}
0 & \Sigma_{1}^{-1} \\
\Sigma_{1}^{-1} & 0
\end{array}\right], \Pi_{2}=\left[\begin{array}{cc}
0 & \Sigma_{2}^{-1} \\
\Sigma_{2}^{-1} & 0
\end{array}\right], \\
\Pi_{3}=\left[\begin{array}{cc}
\bar{\Lambda}_{c}^{-1} & 0 \\
0 & -\bar{\Lambda}_{c}^{-1}
\end{array}\right], \bar{\Sigma}=\operatorname{diag}\left(\Sigma_{1}, \Sigma_{2},\left(\Lambda_{o} \bar{\Lambda}_{c}\right)^{\frac{1}{2}}\right), \\
\bar{\Delta}=\operatorname{diag}\left(s^{-1} I_{d}, s^{-1} I_{n-d}, \tilde{\Delta}\right), \hat{\Delta}=\operatorname{diag}\left(s^{-1} I_{d}, 0_{n-d}, \Delta\right), \\
\bar{M}_{11}=\left[\begin{array}{cc}
\bar{A} & \bar{E} \\
\bar{K} & 0
\end{array}\right], \bar{M}_{12}=\left[\begin{array}{l}
\bar{B} \\
G
\end{array}\right], \bar{M}_{21}=\left[\begin{array}{ll}
\bar{C} & D
\end{array}\right], \bar{M}_{22}=0 .
\end{gathered}
$$

It is easy to check that (20) and (21) are equivalent to (14b) and (15b) respectively. Therefore, the error bound (22) holds from Lemma 13. 


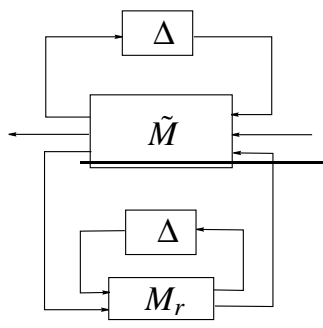

(a) Original configuration.

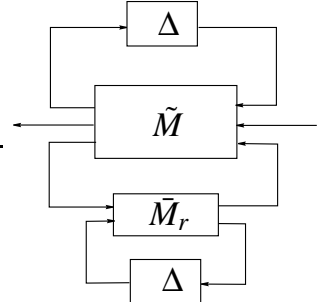

(b) Equivalent configuration.
Fig. 1. LFT configuration.

Note that the above result provides an upper bound on the Hausdorff distance, as defined below, between these two uncertain model sets.

Definition 15: The Hausdorff distance $d_{H}(\mathcal{F}, \mathcal{H})$ between the model sets $\mathcal{F}$ and $\mathcal{H}$ is defined as follows.

$$
\begin{aligned}
d_{H}(\mathcal{F}, \mathcal{H}) & :=\max (\vec{d}(\mathcal{F}, \mathcal{H}), \vec{d}(\mathcal{H}, \mathcal{F})), \\
\vec{d}(\mathcal{F}, \mathcal{H}) & :=\sup _{f(s) \in \mathcal{F}} \inf _{h(s) \in \mathcal{H}}\|f(s)-h(s)\|_{\infty} .
\end{aligned}
$$

If we denote $\mathcal{G}_{\Delta}:=\left\{\mathcal{G}_{\Delta}: \Delta \in \boldsymbol{\Delta}^{\boldsymbol{c}}\right\}$ and $\mathcal{G}_{r \Delta}:=\left\{\mathcal{G}_{r \Delta}: \Delta \in \Delta^{\boldsymbol{c}}\right\}$, the Hausdorff distance between the original system and the reduced system satisfies the following upper bound:

$$
d_{H}\left(\mathcal{G}_{\Delta}, \mathcal{G}_{r \Delta}\right) \leq 2\left(\gamma_{1}^{t}+\cdots+\gamma_{l}^{t}+\bar{\theta}\right)
$$

\section{V. $H_{\infty}$ Model Reduction}

As shown in [26, Theorem 4.20], for a nominal system without uncertainties, generalized Gramians can also be used to characterize the $H_{\infty}$ model reduction problem; see also the original paper [27] and reference [28]. This is true for our uncertain system (1) as well, which is stated as follows.

Theorem 16: Given a robustly stable uncertain system (1), there exists $M_{r}=\left[\begin{array}{c|cc}A_{r} & E_{r} & B_{r} \\ \hline K_{r} & D_{r 11} & D_{r 12} \\ C_{r} & D_{r 21} & D_{r 22}\end{array}\right]$ of order $d$ such that

$$
\sup _{\Delta \in \Delta^{c}}\left\|\mathcal{F}_{u}(M, \Delta)-\mathcal{F}_{u}\left(M_{r}, \Delta\right)\right\|_{\infty}<\varepsilon
$$

if there exist $S>0, P>0, \Lambda_{c} \in \boldsymbol{P}_{\boldsymbol{\Theta}}, \Lambda_{o} \in \boldsymbol{P}_{\boldsymbol{\Theta}}$ solving Riccati inequalities (7), (8) and

$$
\Lambda_{o} \geq \varepsilon^{2} \Lambda_{c}, \lambda_{\text {min }}(S P)=\varepsilon^{2}, \operatorname{rank}\left(S P-\varepsilon^{2} I_{n}\right) \leq d .
$$

Proof: Define an augmented system $\tilde{M}=$ $\left[\begin{array}{ccc}M_{11} & M_{12} & 0 \\ M_{21} & M_{22} & -I \\ 0 & I & 0\end{array}\right]=\left[\begin{array}{c|ccc}A & E & B & 0 \\ \hline K & 0 & G & 0 \\ C & D & 0 & -I \\ 0 & 0 & I & 0\end{array}\right]$, and let $M_{r}=$ $\left[\begin{array}{ll}M_{r 11} & M_{r 12} \\ M_{r 21} & M_{r 22}\end{array}\right]$. Then the overall LFT interconnection of the error system $\mathcal{F}_{u}(M, \Delta)-\mathcal{F}_{u}\left(M_{r}, \Delta\right)$ is shown in Fig. 1(a), which is equivalent to the one in Fig. 1(b) for $\bar{M}_{r}=\left[\begin{array}{ll}M_{r 22} & M_{r 21} \\ M_{r 12} & M_{r 11}\end{array}\right]$. Now our model reduction problem is transformed into an LPV $H_{\infty}$ synthesis problem [12]. Therefore the above results can be proved by using Theorem 5.1 of [12]. Note that in [12] $\|\Delta\|<\varepsilon^{-1}$ is required rather than $\|\Delta\|<1$ in this paper, and thus a corresponding adjustment is needed. (a) Balanced truncation

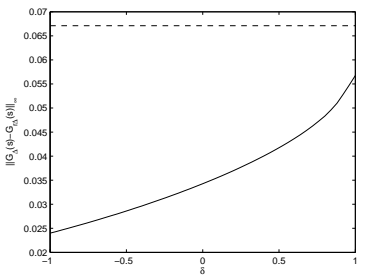

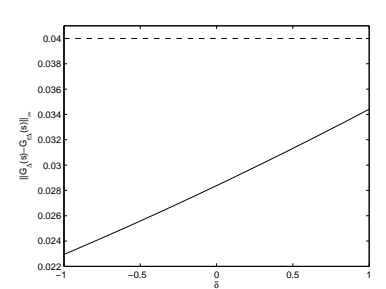

(b) $H_{\infty}$ model reduction
Fig. 2. $H_{\infty}$-norm of the error system as a function of $\delta$.

Remark 1: Unfortunately, the conditions (7), (8) and (23) are not jointly convex; indeed, they are equivalent to (14b), (15b) and the conditions

$$
\left[\begin{array}{cc}
\Lambda_{o} & \varepsilon I_{h} \\
\star & \bar{\Lambda}_{c}
\end{array}\right] \geq 0,\left[\begin{array}{cc}
S & \varepsilon I_{n} \\
\star & P
\end{array}\right] \geq 0, \operatorname{rank}\left[\begin{array}{cc}
S & \varepsilon I_{n} \\
\star & P
\end{array}\right] \leq n+d,
$$

which are rank constrained LMIs. In general, there is no systematic way to solve such problems. However, some of the currently available methods, such as LMIRank [29], perform well in practice. The reduced order model construction follows in a similar way to that in [12] and therefore is omitted here.

\section{EXAMPLE}

Consider the following uncertain system of the form (1) with $\Delta=\delta \in[-1,1]$, and

$$
\begin{gathered}
A=\left[\begin{array}{ccc}
-1 & 0 & 0 \\
1 & -2 & 0 \\
0 & 1 & -3
\end{array}\right], \quad E=\left[\begin{array}{l}
0.1 \\
0.1 \\
0.1
\end{array}\right], \quad B=\left[\begin{array}{l}
1 \\
1 \\
1
\end{array}\right], \\
K=\left[\begin{array}{lll}
1 & 1 & 1
\end{array}\right], \quad C=\left[\begin{array}{lll}
1 & 1 & 1
\end{array}\right], \quad G=1, \quad D=0.1 .
\end{gathered}
$$

Solving the SDP problems (14) and (15), we obtain $\bar{\Lambda}_{c}=$ $32.8993, \Lambda_{o}=0.3469$, and the resulting Gramians are

$$
S=\left[\begin{array}{lll}
0.8327 & 0.8325 & 0.6092 \\
0.8325 & 0.8327 & 0.6092 \\
0.6092 & 0.6092 & 0.4621
\end{array}\right], \quad P=\left[\begin{array}{lll}
1.8721 & 0.9252 & 0.4918 \\
0.9252 & 0.5746 & 0.3540 \\
0.4918 & 0.3540 & 0.2466
\end{array}\right] \text {. }
$$

Then the balanced Gramian is

$$
\Sigma=\operatorname{diag}(2.1728,0.0319,0.0017) \text {. }
$$

Given these values, a natural choice in model reduction would be to truncate the last 2 states. Then, the reduced dimension uncertain system model is defined by

$$
\begin{gathered}
\bar{A}_{r}=-1.0852, \quad \bar{E}_{r}=-0.1693, \quad \bar{B}_{r}=-1.6934, \\
\bar{K}_{r}=-1.6926, \quad \bar{C}_{r}=-1.6926,
\end{gathered}
$$

and the upper bound on the error system is given by

$$
\sup _{\delta \in[-1,1]}\left\|\mathcal{G}_{\Delta}(s)-\mathcal{G}_{r \Delta}(s)\right\|_{\infty} \leq 2(0.0319+0.0017)=0.0672 \text {. }
$$

Figure 2(a) shows $H_{\infty}$-norm of the error system as a function of $\delta$, and the dashed line indicates the bound in (26).

If different uncertainties are allowed, letting $\Delta_{1}=\delta_{1}, \delta_{1} \in$ $[-1,1]$, the upper bound on the error system is given by

$$
\begin{aligned}
\sup _{\delta, \delta_{1} \in[-1,1]}\left\|\mathcal{G}_{\Delta_{1}}(s)-\mathcal{G}_{r \Delta}(s)\right\|_{\infty} \leq 2(0.0319 \\
\quad+0.0017+0.3469 \times 32.8993)=22.8896 .
\end{aligned}
$$




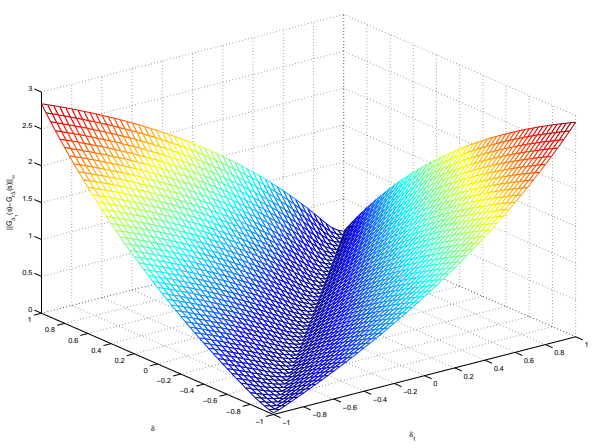

Fig. 3. $H_{\infty}$-norm of the error system as a function of $\delta, \delta_{1}$.

Figure 3 shows $H_{\infty}$-norm of the error system as a function of $\delta, \delta_{1}$, which is less than the bound in (27).

Now we apply the $H_{\infty}$ model reduction algorithm in Section V to the above uncertain system (25). The LMIRank solver [29] is used to solve the rank constrained LMI problem (14b), (15b) and (24). For $\varepsilon=0.04$, we obtain

$$
\begin{gathered}
S=\left[\begin{array}{lll}
0.8367 & 0.8246 & 0.6139 \\
0.8246 & 0.8527 & 0.5951 \\
0.6139 & 0.5951 & 0.4736
\end{array}\right], \quad P=\left[\begin{array}{lll}
1.8734 & 0.9207 & 0.4968 \\
0.9207 & 0.5897 & 0.3377 \\
0.4968 & 0.3377 & 0.2635
\end{array}\right], \\
\bar{\Lambda}_{c}=32.9027, \quad \Lambda_{o}=0.3470 .
\end{gathered}
$$

Then, following a routine similar to that in [12], the reduced dimension uncertain system model is defined by

$$
\begin{aligned}
A_{r} & =-1.0502, \quad E_{r}=0.0118, \quad B_{r}=-1.7315, \\
K_{r} & =23.4812, \quad C_{r}=-1.5945, \quad D_{r 11}=0.0028, \\
D_{r 12} & =-15.1431, \quad D_{r 21}=-0.0070, \quad D_{r 22}=0.0283 .
\end{aligned}
$$

Figure 2(b) shows $H_{\infty}$-norm of the error system as a function of $\delta$, and the dashed line indicates the bound $\varepsilon=0.04$.

\section{CONCLUSIONS}

In this paper a Gramian-based approach to model reduction for a class of uncertain systems with norm bounded structured uncertainty is presented. We introduce notions of controllability and observability Gramians in terms of certain parameterized algebraic Riccati inequalities. This enables us to develop a balanced truncation model reduction method for uncertain systems. Our proposed method is based on the use of LMIs to construct the desired reduced dimension uncertain system model. Error bounds for this model reduction procedure are also derived. $H_{\infty}$ model reduction for uncertain systems is also investigated. A sufficient condition for the existence of a reduced model is provided which involves the underlying Gramians with a rank constraint.

\section{REFERENCES}

[1] B. Moore, "Principal component analysis in linear systems: controllability, observability, and model reduction," IEEE Transactions on Automatic Control, vol. 26, pp. 17-32, 1981.

[2] L. Pernebo and L. Silverman, "Model reduction via balanced state space representations," IEEE Trans. Automat. Contr., vol. 27, no. 2, pp. 382-387, 1982.

[3] K. Glover, "All optimal Hankel-norm approximations of linear multivariable systems and their $L_{\infty}$ error bounds," International Journal of Control, vol. 39, pp. 1115-1193, 1984.
[4] D. Enns, "Model reduction with balanced realizations: an error bound and frequency weighted generalization."

[5] K. Zhou, J. Doyle, and K. Glover, Robust and Optimal Control. Upper Saddle River, NJ: Prentice-Hall, 1996.

[6] I. R. Petersen, V. Ugrinovski, and A. V. Savkin, Robust Control Design using $H^{\infty}$ Methods. Springer-Verlag London, 2000.

[7] W. Wang, J. Doyle, C. Beck, and K. Glover, "Model reduction of LFT systems," in IEEE Conference on Decision and Control, vol. 2, 1991, pp. $1233-1238$.

[8] C. Beck, J. Doyle, and K. Glover, "Model reduction of multidimensional and uncertain systems," IEEE Transactions on Automatic Control, vol. 41, no. 10, pp. 1466-1477, 1996.

[9] S. Lall and C. Beck, "Error-bounds for balanced model-reduction of linear time-varying systems," IEEE Transactions on Automatic Control, vol. 48, no. 6, pp. 946-956, 2003.

[10] H. Sandberg and A. Rantzer, "Balanced truncation of linear timevarying systems," IEEE Transactions on Automatic Control, vol. 49, no. 2, pp. 217-229, 2004.

[11] Y.-C. Chu and K. Glover, "Bounds of the induced norm and model reduction errors for systems with repeated scalar nonlinearities," IEEE Transactions on Automatic Control, vol. 44, no. 3, pp. 471-483, 1999.

[12] P. Apkarian and P. Gahinet, "A convex characterization of gainscheduled $H_{\infty}$ controllers," IEEE Transactions on Automatic Control, vol. 40, no. 5, pp. 853-864, 1995.

[13] G. Scorletti and L. E. Ghaoui, "Improved LMI conditions for gain scheduling and related control problems," International Journal of Robust and Nonlinear Control, vol. 8, no. 10, pp. 845-877, 1998.

[14] P. J. Goddard, Performance-preserving controller approximation. PhD thesis, Cambridge University, 1995.

[15] G. Wood, P. Goddard, and K. Glover, "Approximation of linear parameter-varying systems," in the 35th IEEE Conference on Decision and Control, vol. 1, 1996, pp. 406-411.

[16] F. Wu, "Induced $\mathcal{L}_{2}$ norm model reduction of polytopic uncertain linear systems," Automatica, vol. 32, no. 10, pp. 1417-1426, 1996.

[17] W. Haddad and V. Kapila, "Robust, reduced-order modeling for statespace systems via parameter-dependent bounding functions," IEEE Transactions on Automatic Control, vol. 42, no. 2, pp. 248-253, 1997.

[18] L. Andersson, A. Rantzer, and C. Beck, "Model comparison and simplification," International Journal of Robust and Nonlinear Control, vol. 9, no. 3, pp. 157-181, 1999.

[19] L. Andersson and A. Rantzer, "Frequency-dependent error bounds for uncertain linear models," IEEE Transactions on Automatic Control, vol. 44, no. 11, pp. 2094-2098, 1999.

[20] I. R. Petersen, "Robust unobservability for uncertain linear systems with structured uncertainty," in the 43rd Conference on Decision and Control, vol. 3, Bahamas, December 2004, pp. 2694-2699.

[21] _ "A notion of controllability for uncertain linear systems with structured uncertainty," in the 44th IEEE Conference on Decision and Control, Seville, Spain, December 2005, pp. 2922-2927.

[22] _ "Notions of observability for uncertain linear systems with structured uncertainty," SIAM Journal on Control and Optimization, vol. 41, no. 2, pp. 345-361, 2002.

[23] C. Beck, Model reduction and minimality for uncertain systems. $\mathrm{PhD}$ dissertation, California Inst. Technol., Jan. 1996.

[24] C. Beck and J. Doyle, "A necessary and sufficient minimality condition for uncertain systems," IEEE Transactions on Automatic Control, vol. 44, no. 10 , pp. $1802-1813,1999$.

[25] C. Beck and R. D'Andrea, "Noncommuting multidimensional realization theory: minimality, reachability, and observability," IEEE Transactions on Automatic Control, vol. 49, no. 10, pp. 1815-1822, 2004.

[26] G. E. Dullerud and F. Paganini, A Course in Robust Control Theory : a Convex Approach. New York: Springer, 2000.

[27] D. Kavranoglu and M. Bettayeb, "Characterization of the solution to the optimal $H_{\infty}$ model reduction problem," Systems \& Control Letters, vol. 20, no. 2, pp. 99-107, 1993.

[28] K. M. Grigoriadis, "Optimal $H_{\infty}$ model reduction via linear matrix inequalities: continuous- and discrete-time cases," Systems \& Control Letters, vol. 26, no. 5, pp. 321-333, 1995.

[29] R. Orsi., "LMIRank: software for rank constrained LMI problems, 2005," Available from http://rsise.anu.edu.au/ robert/lmirank/. 